\title{
Ocorrência de formas aladas de pulgões e sua relação com fatores meteorológicos e plantas hospedeiras
}

\author{
Francisco Jorge Cividanes $^{(1)}$ e Terezinha Monteiro dos Santos-Cividanes ${ }^{(2)}$
}

\begin{abstract}
(1)Universidade Estadual Paulista, Faculdade de Ciências Agrárias e Veterinárias, Departamento de Fitossanidade, Via de Acesso Prof. Paulo Donato Castellane, s/no, CEP 14884-900 Jaboticabal, SP. E-mail: fjcivida@fcav.unesp.br (2)Agência Paulista de Tecnologia dos Agronegócios, Avenida Bandeirantes, № 2.419, Vila Virgínia, CEP 14030-670 Ribeirão Preto, SP. E-mail: terezinha@apta.sp.gov.br
\end{abstract}

Resumo - O objetivo deste trabalho foi determinar o padrão de revoadas e a influência de fatores meteorológicos e de Brassicaceae sobre populações de formas aladas dos pulgões Brevicoryne brassicae, Lipaphis erysimi $\mathrm{e}$ Myzus persicae. Os pulgões foram amostrados em armadilhas tipo bandeja amarela com água, entre julho de 1997 e agosto de 2005. A correlação parcial de Pearson foi utilizada para verificar a influência da temperatura do ar, umidade relativa, chuva e insolação na abundância de alados das três espécies. A influência de brassicáceas foi avaliada pelo cálculo do número de graus-dia acumulados acima da temperatura base inferior dessas plantas. Lipaphis erysimi foi mais numerosa que M. persicae e $B$. brassicae. Os alados de B. brassicae apresentaram revoadas que predominaram de agosto a outubro, com o pico de abundância em setembro. Os períodos de revoada de L. erysimi e M. persicae foram mais longos que o de B. brassicae, com os maiores picos de L. erysimi e M. persicae observados de abril a novembro e de junho a outubro, respectivamente. A população de $B$. brassicae teve correlação significativa com as temperaturas máxima e mínima, insolação e umidade relativa do ar, enquanto L. erysimi e $M$. persicae foram afetadas apenas pela insolação e umidade relativa.

Termos para indexação: Brassica oleracea, dinâmica populacional, dispersão, padrão de revoada, pulgão.

\section{Occurrence of alate aphids and their relationship with meteorological factors and host plants}

\begin{abstract}
The objective of this work was to determine the flight patterns and the influence of meteorological factors and Brassicaceae on the populations of the alate aphids Brevicoryne brassicae, Lipaphis erysimi and Myzus persicae. The alate aphids were sampled using yellow water traps between July of 1997 and August of 2005. The Pearson partial correlation was used to determine the influence of air temperature, relative humidity, rainfall and insolation on the abundance of alate. The influence of Brassicaceae was assessed by accumulated degree-days above the temperature threshold of these plants. Lipaphis erysimi was more abundant than M. persicae and B. brassicae. The alate B. brassicae showed the highest flight frequency between August and October, with the abundance peak in September. The flight frequency of $L$. erysimi and $M$. persicae were longer than B. brassicae, with the alate abundance peaks of L. erysimi and M. persicae observed from April to November and from June to October, respectively. The population of $B$. brassicae showed significant correlation with the maximum and minimum temperatures, insolation and relative humidity, while L. erysimi and M. persicae were only affected by insolation and relative humidity.
\end{abstract}

Index terms: Brassica oleracea, population dynamics, dispersion, flight pattern, aphid.

\section{Introdução}

Os pulgões caracterizam-se por apresentar inúmeros tipos de associações com plantas hospedeiras, intrincados ciclos de vida, polimorfismo e habilidade para se reproduzir assexuada e sexuadamente (Williams \& Dixon, 2007).

De acordo com Kuo (1999), quanto maior a densidade populacional dos pulgões, maior a tendência de que os descendentes sejam alados. No entanto, vários outros fatores são reconhecidos como influentes no aparecimento de formas aladas nas populações de pulgões, em que se destacam: a temperatura, o fotoperíodo, as mudanças na qualidade nutricional da planta hospedeira (Hodgson et al., 2005; Aheer et al., 2008; Mirmohammadi et al., 2009) e a presença de inimigos naturais (Pereira \& Lomônaco, 2003). Com relação à temperatura, Bale et al. (2007) 
relataram que esse fator pode influenciar o voo de pulgões. Os autores ainda mencionaram que a baixa temperatura, apesar de suprimir o desenvolvimento de asas nos pulgões, pode aumentar a fecundidade desses insetos e contribuir para a dispersão e migração de alados, fatores considerados importantes para o sucesso dos pulgões como grupo.

Kindlmann et al. (2007) destacaram a necessidade do entendimento da dinâmica populacional dos pulgões, para o adequado manejo de suas populações. Contudo, mencionaram não ser uma tarefa fácil o conhecimento dessa dinâmica para propósitos práticos, em razão de ocorrerem oscilações na densidade dos pulgões, causadas por fatores intrínsecos à população (fecundidade, mortalidade, taxa de migração) e extrínsecos (condições meteorológicas, especialmente temperatura, e qualidade da planta hospedeira). Chattopadhyay et al. (2005) indicaram as condições meteorológicas como fatores importantes para a dinâmica populacional de pulgões.

Os pulgões Brevicoryne brassicae (L.), Lipaphis erysimi (Kalt.) e Myzus persicae (Sulzer) encontram-se distribuídos mundialmente e podem causar danos a diversas culturas, além de transmitir vírus causadores de doenças às plantas (Emden \& Harrington, 2007). No Brasil, há poucos trabalhos sobre a dinâmica populacional de formas aladas de B. brassicae, L. erysimi e M. persicae. Costa (1970) estudou a migração de $M$. persicae na região de Campinas, SP. Alguns estudos relacionam-se com levantamento populacional dos alados das referidas espécies (Oliveira, 1971; Rossi et al., 1990; Carvalho et al., 2002; Cividanes, 2002a).

O objetivo deste trabalho foi determinar o padrão de revoadas e a influência de fatores meteorológicos e de Brassicaceae sobre populações de formas aladas dos pulgões B. brassicae, L. erysimi e M. persicae.

\section{Material e Métodos}

O estudo foi conduzido no campus da Faculdade de Ciências Agrárias e Veterinárias (FCAV), da Universidade Estadual Paulista (Unesp), Jaboticabal, SP, de julho de 1997 a agosto de 2005. O clima local é classificado segundo Köppen, como Cwa, subtropical, mesotérmico, com inverno seco, verão chuvoso, temperatura do mês mais quente superior a $22^{\circ} \mathrm{C}$ e temperatura do mês mais frio inferior a $18{ }^{\circ} \mathrm{C}$. O solo da área experimental é classificado como Latossolo Vermelho-Escuro e estava cultivado com uma coleção de plantas forrageiras, em que predominava alfafa (Medicago sativa L.). A área estava a pelo menos $200 \mathrm{~m}$ de distância de plantações de couve-de-folha (Brassica oleracea L. var. acephala DC.) e de repolho (Brassica oleracea L. var. capitata L.).

Olevantamento populacional dos pulgões foirealizado com uso de duas armadilhas tipo bandeja amarela com água, distanciadas $10 \mathrm{~m}$ entre si e inseridas em aros de metal, suspensos por hastes de madeira a $120 \mathrm{~cm}$ de altura em relação ao solo, conforme metodologia utilizada por Gutierrez et al. (1974). Foram utilizadas armadilhas com $36 \mathrm{~cm}$ de diâmetro e $15 \mathrm{~cm}$ de altura, com dois orifícios laterais vedados com malha de plástico, para evitar transbordamento nos períodos chuvosos, e pintadas internamente com tinta amarelo-brilhante Wandalux (Akzo Nobel, São Bernardo do Campo, Brasil), e, externamente, com tinta verde-colonial acetinado Suvinil (Suvinil, São Bernardo do Campo, Brasil). Dentro das armadilhas foram colocados $12 \mathrm{~L}$ de água, com $5 \mathrm{~mL}$ de detergente neutro e $50 \mathrm{~mL}$ de formol, para diminuir a tensão superficial da água e melhor conservar os insetos capturados, respectivamente.

Os insetos foram retirados semanalmente das armadilhas com peneira de malha ultrafina. No laboratório, os pulgões alados foram separados com o auxílio de uma lupa de mesa com luz e mantidos em frascos com álcool a $80 \%$. Posteriormente, com uso de microscópio estereoscópico, procedeu-se à identificação e à contagem das espécies de pulgões.

Para a determinação do material, foram utilizados, para comparação, exemplares alados e ápteros de $B$. brassicae, L. erysimi e M. persicae. A influência de fatores do ambiente sobre a ocorrência das formas aladas foi avaliada por meio de correlação parcial de Pearson (Rao, 1998), conforme Honek (1985). As variáveis consideradas na análise foram: temperaturas máxima e mínima, umidade relativa, insolação, precipitação pluvial diária e o número de graus-dia acumulados acima de $4^{\circ} \mathrm{C}$, considerada a temperatura base inferior de brassicáceas (González Benavente-Garcia et al., 1993). Os fatores meteorológicos foram registrados na Estação Agroclimatológica da FCAV/Unesp $\left(21^{\circ} 14^{\prime} 05^{\prime \prime} \mathrm{S}\right.$, $48^{\circ} 17^{\prime} 09^{\prime \prime} \mathrm{W}$ e altitude de $615 \mathrm{~m}$ ), distante cerca de $500 \mathrm{~m}$ das armadilhas.

$\mathrm{Na}$ análise, considerou-se o número total de formas aladas capturadas de cada espécie, nas duas 
armadilhas. A soma de graus-dia foi iniciada a partir de $1^{\circ}$ de janeiro de cada ano estudado, tendose considerado a soma acumulada de graus-dia até cada data da retirada dos pulgões das armadilhas. Os fatores meteorológicos temperatura, umidade relativa e insolação foram representados pela média dos valores registrados no período dos sete dias anteriores à data da retirada dos pulgões das armadilhas. Para a precipitação pluvial, considerou-se a soma registrada no referido período.

Com base em dados de Cividanes (2002b), determinou-se a regressão linear entre a taxa de mortalidade diária de $B$. brassicae, em plantas de couve, e a média das temperaturas mínimas dos sete dias anteriores ao dia da constatação da mortalidade, entre 8 e 23 de maio de 1999.

\section{Resultados e Discussão}

Entre as três espécies de pulgões, $L$. erysimi foi a mais numerosa, com 2.197 indivíduos capturados que representaram $68,8 \%$ do total de indivíduos observados no trabalho. A segunda espécie em abundância foi M. persicae, seguida por B. brassicae, das quais foram capturados $692(21,7 \%)$ e 303 indivíduos (9,5\%), respectivamente.

Os períodos de revoadas de $B$. brassicae predominaram de agosto até outubro, na maioria dos anos estudados, com ocorrência de pico de abundância geralmente em setembro (Figura 1). Lipaphis erysimi e M. persicae apresentaram revoadas durante períodos mais longos que $B$. brassicae, com destaque para L. erysimi, cujos alados estiveram presentes praticamente durante todo o ano (Figuras 2 e 3). Os maiores picos de abundância de $L$. erysimi ocorreram entre abril e novembro, e os de $M$. persicae de junho a outubro, com vários desses picos observados no mês de setembro para ambas as espécies.

De modo geral, as informações sobre ecologia de pulgões referem-se a espécies presentes em regiões de clima temperado do Hemisfério Norte, com poucos estudos na América do Sul, África e Índia. As informações sobre $M$. persicae indicam que é uma espécie altamente polífaga (generalista), o que, aliado ao ciclo de vida, possibilita a sua ocorrência durante quase todo o ano, em condições de clima temperado (Blackman \& Eastop, 2000; Blande et al., 2004; Emden \& Harrington, 2007). Lipaphis erysimi, apesar de ocorrer em brassicáceas (Blande et al., 2004), é também encontrada em outras espécies de plantas como o agrião (Blackman \& Eastop, 2000), a batata (Carrera \& Cermeli, 2001) e o tomate (Nasreen et al., 2004). Brevicoryne brassicae difere das duas espécies anteriores, por ser considerada especialista quanto ao hábito alimentar, com plantas hospedeiras que são quase exclusivamente da família Brassicaceae (Pontoppidan et al., 2003).

Portanto, os resultados do presente trabalho, que indicam revoadas de $B$. brassicae em período mais restrito do ano que as revoadas de $M$. persicae e L. erysimi (Figuras 1 a 3), devem estar relacionados em grande parte à presença das plantas hospedeiras. Em B. brassicae, as revoadas (agosto a outubro) coincidiram, em grande parte, com o período de junho a setembro, que concentra o cultivo de brassicáceas na região de Jaboticabal.

Os alados de B. brassicae foram os que mais interagiram significativamente com os fatores físicos do ambiente, tendo apresentado correlação positiva com a temperatura máxima, e negativa com a temperatura mínima, com a insolação e com a umidade relativa do ar (Tabela 1). As espécies L. erysimi e M. persicae foram afetadas apenas pela insolação e pela umidade relativa, com as quais se correlacionaram do mesmo modo como citado anteriormente para $B$. brassicae. Ressalta-se que a insolação e a umidade relativa atuaram negativa e significativamente sobre as três espécies, o que indica que o número de alados presentes no ar diminuiu com o aumento desses fatores. Os resultados obtidos para B. brassicae coincidem parcialmente com os de outros estudos (Rossi et al., 1990; Cividanes, 2002a), diferem, contudo, quanto a L. erysimi e $M$. persicae (Rossi et al., 1990). As diferenças encontradas se devem, provavelmente, ao fato de que o padrão da variação da densidade populacional de determinada espécie de pulgão depende de inúmeros fatores (Kindlmann et al., 2007), o que pode contribuir para que ocorram diferenças na dinâmica populacional de uma mesma espécie localizada em regiões distintas, além de diferenças relacionadas às metodologias da análise dos dados utilizadas nos diferentes estudos.

De acordo com Honek (1985), espécies de pulgões influenciadas de modo semelhante por fatores meteorológicos apresentam características similares em abundância e preferência por microclima. Assim, 

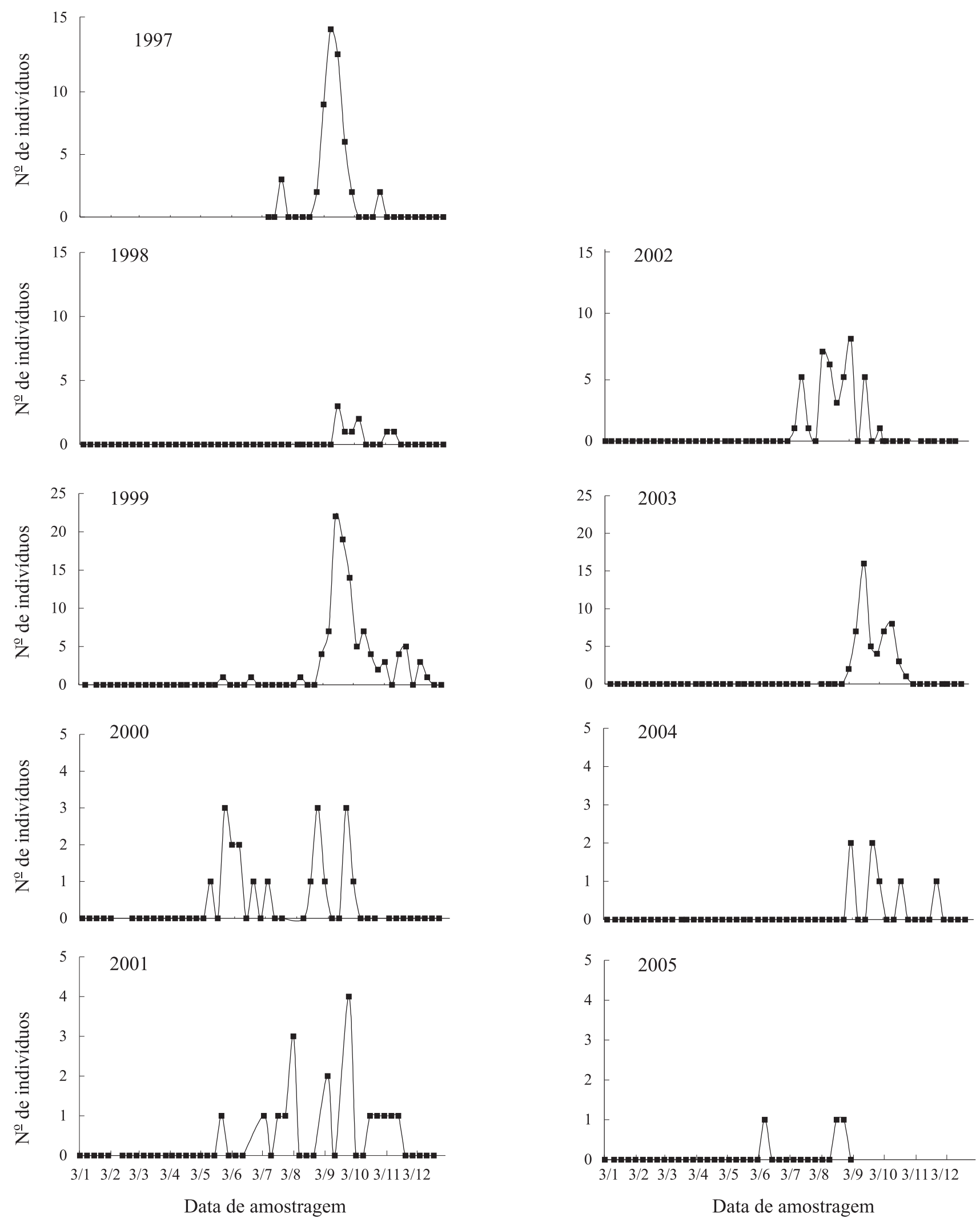

Figura 1. Flutuação na abundância de formas aladas de Brevicoryne brassicae, em Jaboticabal, SP, de 7/1997 a 8/2005. 

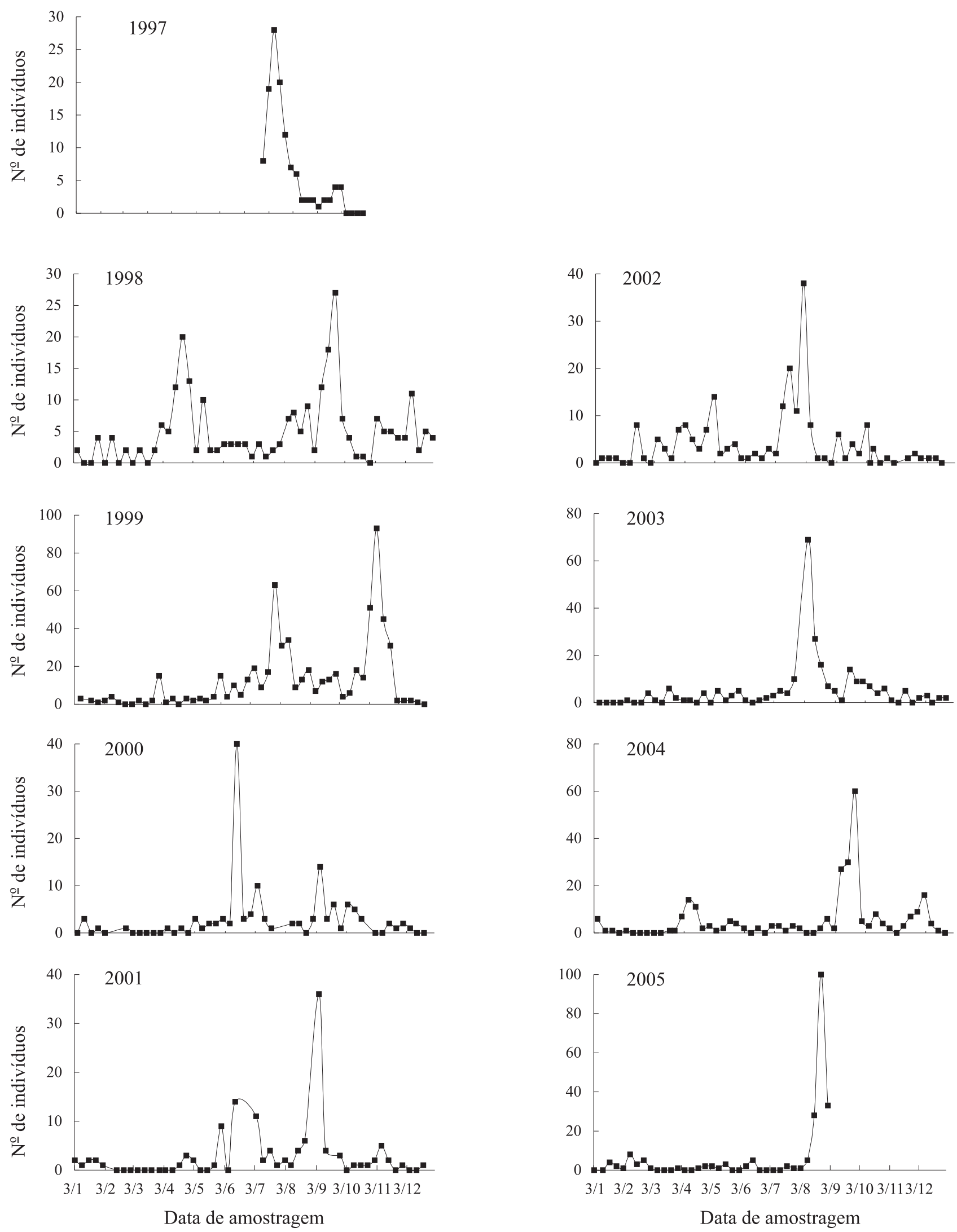

Figura 2. Flutuação na abundância de formas aladas de Lipaphis erysimi, em Jaboticabal, SP, de 7/1997 a 8/2005. 

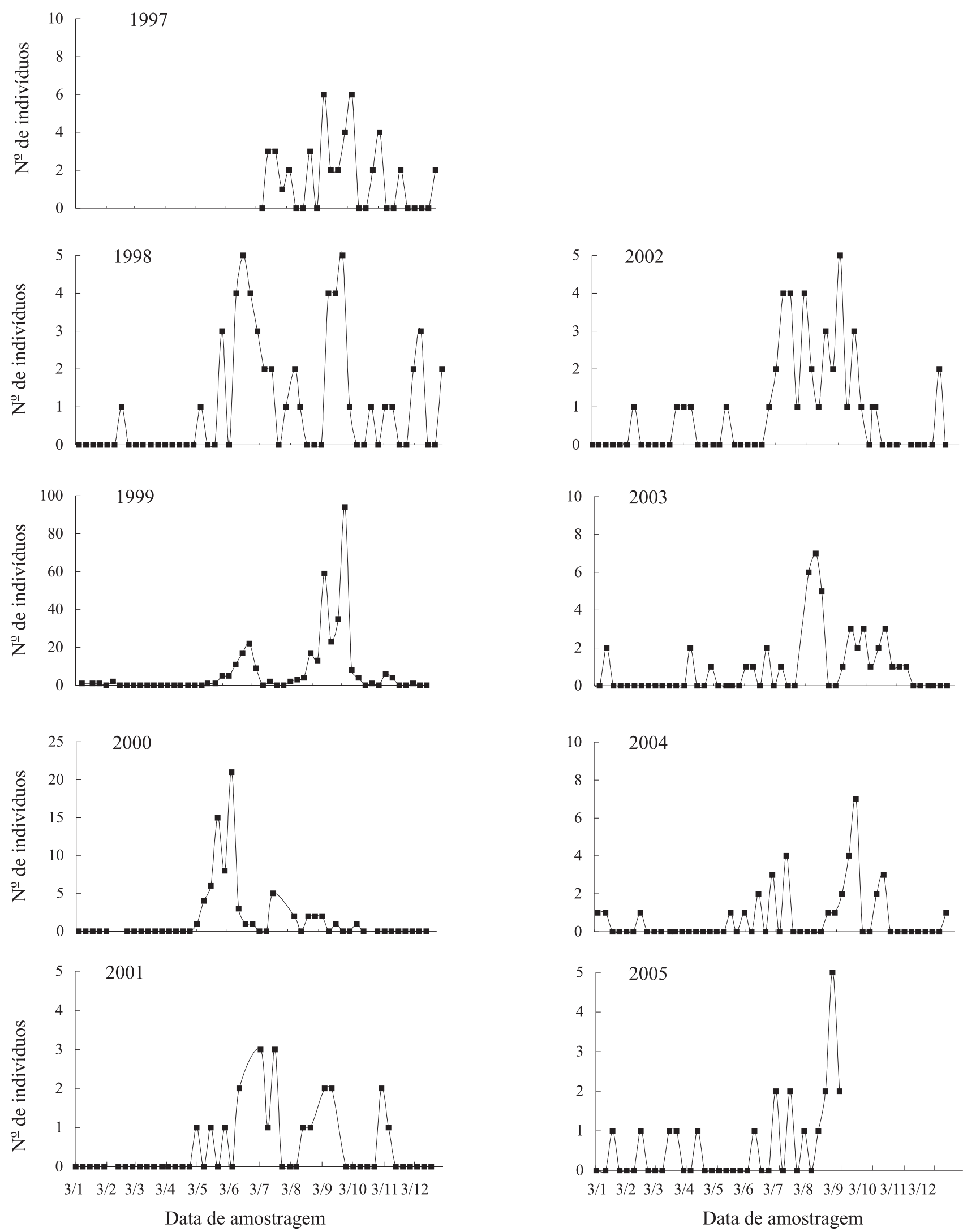

Figura 3. Flutuação na abundância de formas aladas de Myzus persicae, em Jaboticabal, SP, de 7/1997 a 8/2005. 
os resultados do presente trabalho indicam que a dinâmica de alados de $B$. brassicae difere da dinâmica dos alados de L. erysimi e M. persicae, as quais se mostraram similares entre si.

A correlação positiva e significativa entre a variação da densidade de $B$. brassicae e a temperatura máxima (Tabela 1) indica que as revoadas de $B$. brassicae aumentaram com o aumento da temperatura. Ressalta-se que as brassicáceas são hidricamente exigentes e podem ser mais afetadas pelo estresse hídrico em períodos de temperaturas elevadas (Filgueira, 2000). Portanto, a correlação positiva entre a temperatura máxima do ambiente e a abundância de $B$. brassicae pode estar relacionada ao surgimento de formas aladas, em razão do estresse causado às brassicáceas pelos períodos de temperaturas elevadas, associados ao deficit hídrico, ocorridos na região de Jaboticabal. Esses resultados encontram respaldo nos estudos de King et al. (2006), que relataram que $B$. brassicae e $L$. erysimi responderam diferentemente, ao serem mantidas em couve-nabiça (Brassica napus L.), sob deficiência hídrica. Nessas condições, B. brassicae apresentou maior número de alados, além de ápteros em baixo número e de menor tamanho, em comparação a $L$. erysimi, que não teve essas características afetadas pela limitação hídrica.

No presente trabalho, o local de amostragem dos pulgões estava a cerca de $200 \mathrm{~m}$ da área experimental do Departamento de Fitossanidade, onde, praticamente durante todo o período do estudo, havia plantas de couve-de-folha ou de repolho frequentemente submetidas à deficiência hídrica, pois ficaram sem receber irrigação quando não utilizadas em

Tabela 1. Coeficientes de correlação parcial da abundância de formas aladas de pulgões e as variáveis ambientais mensuradas: soma acumulada de grau-dia (GD), temperatura máxima (Tmáx), temperatura mínima (Tmín), insolação (Insol), umidade relativa (UR) e precipitação pluvial (Prec).

\begin{tabular}{lccc}
\hline Coeficiente & \multicolumn{3}{c}{ Espécie } \\
\cline { 2 - 4 } & B. brassicae & L. erysimi & M. persicae \\
\hline GD & 0,0474 & 0,0202 & $-0,0034$ \\
Tmáx $\left({ }^{\circ} \mathrm{C}\right)$ & $0,1372^{* *}$ & 0,0564 & 0,0347 \\
Tmín $\left({ }^{\circ} \mathrm{C}\right)$ & $-0,1401^{* *}$ & $-0,0776$ & $-0,0619$ \\
Insol $(\mathrm{h})$ & $-0,2250^{* *}$ & $-0,1383^{* *}$ & $-0,1426^{* *}$ \\
UR $(\%)$ & $-0,1007^{*}$ & $-0,1275^{* *}$ & $-0,0959^{*}$ \\
Prec $(\mathrm{mm})$ & 0,0537 & $-0,0027$ & 0,0094 \\
$\mathrm{n}$ & 409 & 402 & 409 \\
\hline
\end{tabular}

* e **Significativo a 5 e $1 \%$ de probabilidade pelo teste $\mathrm{t}$, respectivamente; $\mathrm{n}$, número de pares de dados. experimentos. Geralmente essas plantas apresentavam infestações de B. brassicae, L. erysimi e M. persicae e podem ter contribuído para o surgimento de alados em consequência do estresse hídrico.

O número de alados de $B$. brassicae (Tabela 1) aumentou com o decréscimo da temperatura mínima do ambiente. A influência de baixas temperaturas sobre a formação de alados de pulgões foi também constatada por Kuo (1999), que indicou gradiente entre 15 e $20^{\circ} \mathrm{C}$ como ótimo para a formação de alados de M. persicae, mas que as temperaturas de 10 e $30^{\circ} \mathrm{C}$ impedem a formação de alados da espécie.

Embora o presente trabalho indique que a ocorrência de alados de $B$. brassicae aumentou com o decréscimo da temperatura mínima, as formas ápteras podem ter taxas de mortalidade maiores com o declínio da temperatura mínima do ambiente (Figura 4). Em estudo realizado no outono de 1999, observou-se brusca diminuição na sobrevivência de adultos ápteros de $B$. brassicae, a partir do dia 8 de maio. Os registros de temperatura indicaram queda da temperatura mínima a partir desse dia, que tendeu a diminuir até a morte dos últimos adultos no dia 23 de maio. $\mathrm{O}$ ajuste do modelo de regressão linear foi altamente significativo $\left(\mathrm{F}=210,8^{* *}\right.$; $\mathrm{p}<0,01$ ), tendo evidenciado que a queda da temperatura mínima diminuiu mais rapidamente a sobrevivência de $B$. brassicae naquela época do ano. A média da temperatura mínima, no período em que se observou maior mortalidade dos adultos ápteros do pulgão (8 a $27 \mathrm{de}$ maio), foi de $12^{\circ} \mathrm{C}$. Esses resultados indicam que

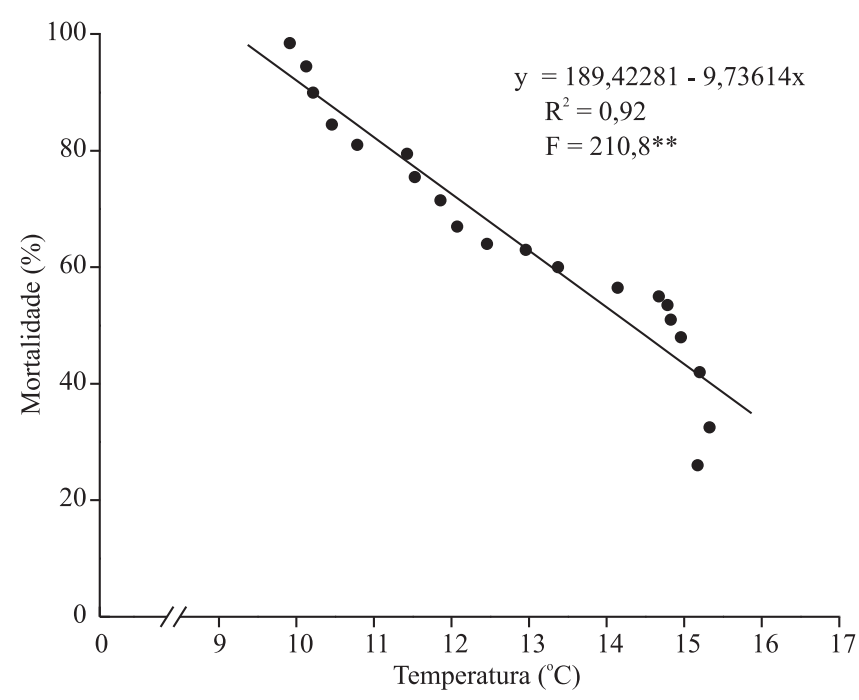

Figura 4. Efeito da temperatura mínima do ar sobre a mortalidade de ápteros de Brevicoryne brassicae, durante o outono de 1999, em Jaboticabal, SP. 
existe uma temperatura mínima limite, a partir da qual a população de ápteros e, consequentemente, de alados da espécie tende a diminuir.

\section{Conclusões}

1. A abundância mais elevada de alados ocorre em setembro, em Brassica brassicae, de abril a novembro, em L. erysimi, e de junho a outubro, para Myzus persicae.

2. Lipaphis erysimi e M. persicae apresentam padrão de revoadas similar, mas que difere de $B$. brassicae.

3. A ocorrência de alados de B. brassicae apresenta correlação positiva e negativa com a temperatura máxima e mínima, respectivamente.

\section{Agradecimentos}

Ao Dr. José Carlos Barbosa e ao Dr. Gener Tadeu Pereira, Departamento de Ciências Exatas da Faculdade de Ciências Agrárias e Veterinárias, da Universidade Estadual Paulista (FCAV/Unesp), pelo auxílio na análise estatística; ao Dr. Carlos Roberto Souza e Silva, Departamento de Ecologia e Biologia Evolutiva, da Universidade Federal de São Carlos, pela identificação das espécies de pulgões; aos Srs. Alex Antonio Ribeiro, José Altamiro de Souza e José Luiz Dacal Seguim, Departamento de Fitossanidade da FCAV/Unesp, pela participação no levantamento em campo; ao Conselho Nacional de Desenvolvimento Científico e Tecnológico, por bolsa concedida.

\section{Referências}

AHEER, G.M.; AMJAD, A.; MANZOOR A. Abiotic factors effect on population fluctuation of alate aphids in wheat. Journal of Agricultural Research, v.46, p.367-371, 2008.

BALE, J.S.; PONDER, K.L.; PRITCHARD, J. Coping with stress. In: EMDEN, H.F. van; HARRINGTON, R. (Ed.). Aphids as crop pests. Wallingford: CAB International, 2007. p.287-309.

BLACKMAN, R.L.; EASTOP, V.F. Aphids on the world's crops: an identification and information guide. $2^{\text {nd }}$ ed. Chichester: John Wiley and Sons, 2000. 476p.

BLANDE, J.D.; PICKETT, J.A.; POPPY, G.M. Attack rate and success of the parasitoid Diaeretiella rapae on specialist and generalist feeding aphids. Journal of Chemical Ecology, v.30, p.1781-1795, 2004.

CARRERA, A.; CERMELI, M. Fluctuation and identification of aphids at three potato (Solanum tuberosum L.) producing localities in Monagas State, between 1987 and 1999. Entomotropica, v.16, p.67-72, 2001.

CARVALHO, L.M.; BUENO, V.H.P.; MARTINEZ, R.P. Levantamento de afídeos alados em plantas hortícolas em Lavras-MG. Ciência e Agrotecnologia, v.26, p.523-532, 2002.

CHATTOPADHYAY, C.; AGRAWAL, R.; KUMAR, A.; SINGH, Y.P.; ROY, S.K.; KHAN, S.A.; BHAR, L.M.; CHAKRAVARTHY, N.V.K.; SRIVASTAVA, A.; PATEL, B.S.; SRIVASTAVA, B.; SINGH, C.P.; MEHTA, S.C. Forecasting of Lipaphis erysimi on oilseed Brassicas in India - a case study. Crop Protection, v.24, p.1042-1053, 2005.

CIVIDANES, F.J. Flutuação populacional de formas aladas de Brevicoryne brassicae (L.) (Hemiptera: Aphididae). Bragantia, v.61, p.143-150, 2002a.

CIVIDANES, F.J. Tabelas de vida de fertilidade de Brevicoryne brassicae (L.) (Hemiptera: Aphidide) em condições de campo. Neotropical Entomology, v.31, p.419-427, 2002b.

COSTA, C.L. Variações sazonais da migração de Myzus persicae em Campinas nos anos de 1967 a 1969. Bragantia, v.29, p.347-360, 1970.

EMDEN, H.F. van; HARRINGTON, R. (Ed.). Aphids as crop pests. Wallingford: CAB International, 2007. 717p.

FILGUEIRA, F.A.R. Novo manual de olericultura: agrotecnologia moderna na produção e comercialização de hortaliças. Viçosa: UFV, 2000. 402p.

GONZÁLEZ BENAVENTE-GARCIA, A.; FERNÁNDEZ HERNANDEZ, J.A.; MUÑOZ FRUCTUOSO, J.; MONTORO DE ANTONIO, P.; PÉREZ CAÑAVATE, J.G. Calendarios de producción de brócoli para otoño-primavera. Agrícola Vergel, agosto, p.423-428, 1993.

GUTIERREZ, A.P.; NIX, H.A.; HAVENSTEIN, D.E.; MOORE, P.A. The ecology of Aphis craccivora Koch and subterranean clover stunt virus in South-East Australia. Journal of Applied Ecology, v.11, p.21-35, 1974.

HODGSON, E.W.; VENETTE, R.C.; ABRAHAMSON, M.; RAGSDALE, D.W. Alate production of soybean aphid (Homoptera: Aphididae) in Minnesota. Environmental Entomology, v.34, p.1456-1463, 2005.

HONEK, A. Temperature and plant vigour influence annual variation of abundance in cereal aphids (Homoptera, Aphididae). Journal of Plant Diseases and Protection, v.92, p.588-593, 1985.

KINDLMANN, P.; JAROSÍK, V.; DIXON, A.F.G. Population dynamics. In: EMDEN, H.F. van; HARRINGTON, R. (Ed.). Aphids as crop pests. Wallingford: CAB International, 2007. p.311-329.

KING, C.; JACOB, H.S.; BERLANDIER, F. The influence of water deficiency on the relationship between canola (Brassica napus L.), and two aphid species (Hemiptera: Aphididae), Lipaphis erysimi (Kaltenbach) and Brevicoryne brassicae (L.). Australian Journal of Agricultural Research, v.57, p.439-445, 2006.

KUO, M.H. Effects of temperature, photoperiod and crowding treatment on alate formation in the turnip aphid, Lipaphis erysimi (Kalt.). Plant Protection Bulletin, v.41, p.255-264, 1999. 
MIRMOHAMMADI, S.; ALLAHYARI, H.; NEMATOLLAHI, M.R.; SABOORI, A. Effect of host plant on biology and life table parameters of Brevicoryne brassicae (Hemiptera: Aphididae). Annals of the Entomological Society of America, v.102, p.450-455, 2009.

NASREEN, A.; MUSTAFA, G.; IQBAL, M.; ULLAH, A. Biologically based management of sucking insect pest of tomato. Pakistan Entomologist, v.26, p.75-79, 2004.

OLIVEIRA, A.M. de. Observações sobre a influência de fatores climáticos nas populações de afídeos em batata. Pesquisa Agropecuária Brasileira, v.6, p.163-172, 1971.

PEREIRA, C.D.; LOMÔNACO, C. Produção de formas aladas em colônias de Brevicoryne brassicae (L.) (Hemiptera: Aphididae) por indução do parasitóide Diaeretiella rapae (McIntosh) (Hymenoptera: Aphidiidae) em alguns aspectos comportamentais da interação destas espécies. Neotropical Entomology, v.32, p.97-102, 2003.
PONTOPPIDAN, B.; HOPKINS, R.; RASK, L.; MEIJER, J. Infestation by cabbage aphid (Brevicoryne brassicae) on oilseed rape (Brassica napus) causes a long lasting induction of the myrosinase system. Entomologia Experimentalis et Applicata, v.109, p.55-62, 2003.

RAO, P.V. Statistical research methods in the life sciences. New York: Duxbury Press, 1998. 889p.

ROSSI, M.M.; MATIOLI, J.C.; CARVALHO, C.F. Efeitos de fatores climáticos sobre algumas espécies de pulgões (Homoptera: Aphididae) na cultura da batata. Anais da Sociedade Entomológica do Brasil, v.19, p.75-86, 1990.

WILLIAMS, I.S.; DIXON, A.F.G. Life cycles and polymorphism. In: EMDEN, H.F. van; HARRINGTON, R. (Ed.). Aphids as crop pests. Wallingford: CAB International, 2007. p.69-85.

$\overline{\text { Recebido em } 23 \text { de novembro de } 2009 \text { e aprovado em } 29 \text { de dezembro de } 2009}$ 